\title{
Effect of magnetic field on quasiparticle branches of intrinsic Josephson junctions with ferromagnetic layer
}

\author{
L. Ozyuzer ${ }^{\mathrm{a}, *}$, M. Ozdemir ${ }^{\text {a }}$, C. Kurter ${ }^{\text {b }}$, D.G. Hinks ${ }^{\text {b }}$, K.E. Gray ${ }^{\text {b }}$ \\ ${ }^{a}$ Department of Physics, Izmir Institute of Technology, TR-35430 Izmir, Turkey \\ b Materials Science Division, Argonne National Laboratory, Argonne, IL 60439, USA
}

Available online 3 April 2007

\begin{abstract}
The interlayer tunneling spectroscopy has been performed on micron-sized mesa arrays of $\mathrm{HgBr}_{2}$ intercalated superconducting $\mathrm{Bi} 2212$ single crystals. A ferromagnetic multilayer $(\mathrm{Au} / \mathrm{Co} / \mathrm{Au})$ is deposited on top of the mesas. The spin-polarized current is driven along the $c$-axis of the mesas through a ferromagnetic Co layer and the hysteretic quasiparticle branches are observed at $4.2 \mathrm{~K}$. Magnetic field evolution of hysteretic quasiparticle branches is obtained to examine the effect of injected spin-polarized current on intrinsic Josephson junction characteristics. It is observed that there is a gradual distribution in quasiparticle branches with the application of magnetic field and increasing field reduces the switching current progressively.
\end{abstract}

(C) 2007 Elsevier B.V. All rights reserved.

Keywords: Intrinsic Josephson junctions; Spin-polarized current; Tunneling spectroscopy

The injection of spin-polarized current through the $c$-axis of Bi2212 can give a deep insight into the mechanism of high temperature superconductors as well as it might open new application areas for spintronics [1]. We report the tunneling characteristics of mesa arrays on $\mathrm{HgBr}_{2}$ intercalated Bi2212 with spin-polarized current. Since heating is one of the biggest challenges for intrinsic Josephson junctions (IJJs) of pure $\mathrm{Bi} 2212, \mathrm{HgBr}_{2}$ intercalated single crystals which have at least 100 times larger $c$-axis resistivity than pristine ones were used for the measurements. Pattering of the intercalated crystals with $T_{\mathrm{c}}=74 \mathrm{~K}$ is essential to obtain IJJ tunneling characteristics. To get one set of the samples, freshly cleaved single crystals were immediately sputtered with a ferromagnetic multilayer $(\mathrm{Au}=15 \mathrm{~nm} /$ $\mathrm{Co}=80 \mathrm{~nm} / \mathrm{Au}=156 \mathrm{~nm}) .10 \times 10 \mu \mathrm{m}^{2}$ mesa arrays were fabricated using photolithography and $\mathrm{Ar}$ ion etching described elsewhere [2]. The heights of the mesas (merely IJJ stacks) were obtained as $73 \mathrm{~nm}$ by AFM.

\footnotetext{
* Corresponding author. Tel.: +90 232750 7518; fax: +90 2327507509 . E-mail address: ozyuzer@iyte.edu.tr (L. Ozyuzer).
}

A three probe technique was used to obtain $I-V$ characteristics at $4.2 \mathrm{~K}$. A sharpened gold wire was mounted to a point contact tunneling assembly, and since the mesa area is micron-sized, third contact was obtained from top of it by this wire. Magnetic field was applied along the $c$-axis of the crystals during the measurements.

Fig. 1a and b shows $I-V$ characteristics of a mesa with ferromagnetic Co layer at $4.2 \mathrm{~K}$ with $B=0$ and $B=$ $200 \mathrm{G}$, respectively. Non-existence of backbending around the sumgap voltage is an indication of less heating in the mesa. The sumgap branch of the mesa does not change in position with applied magnetic field as seen in Fig. 1b. On the other hand, recent IJJ study on pristine Bi2212 with ferromagnetic layer has shown that there is a reduction in Josephson critical current and the superconducting gap with magnetic field [3].

Fig. 2a and $\mathrm{b}$ is the detailed representations of Fig. 1a and $\mathrm{b}$ showing the hysteretic quasiparticle branches. In Fig. 2a, the switching current of quasiparticle branches exhibits a gradual distribution. Since cobalt is not epitaxially grown on $\mathrm{Au}$, the amount of polarization of quasiparticles injected into the high $T_{\mathrm{c}}$ superconductor is not 

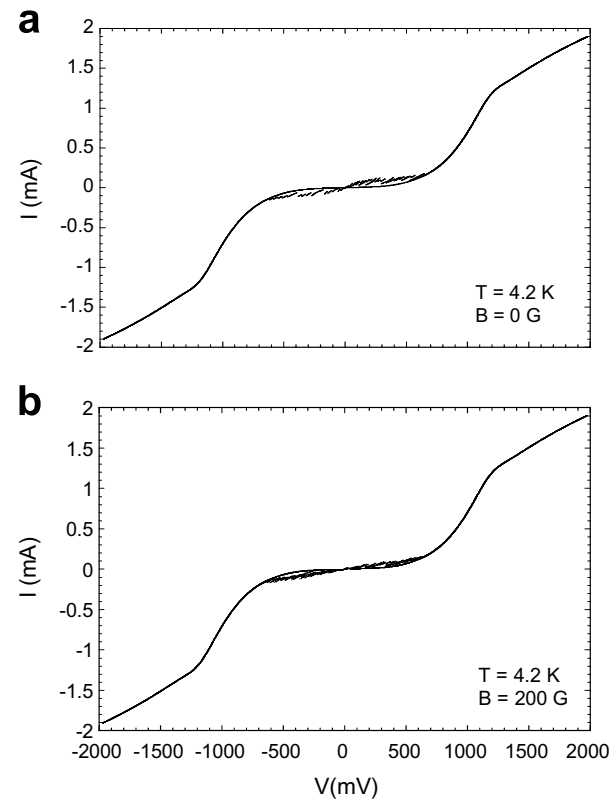

Fig. 1. $I-V$ characteristics of a mesa with cobalt top layer: (a) $B=0 \mathrm{G}$, (b) $B=200 \mathrm{G}$.
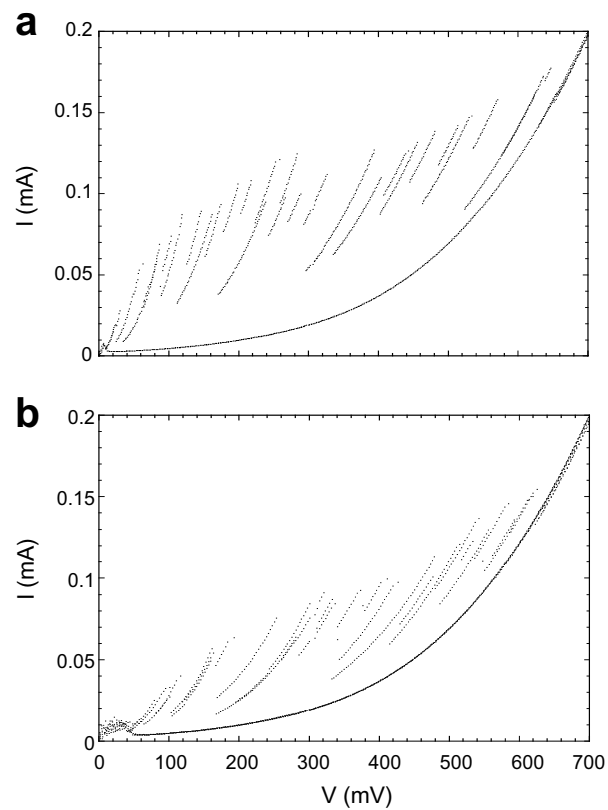

Fig. 2. Detailed $I-V$ characteristics of a mesa given in Fig. 1: (a) $B=0 \mathrm{G}$, (b) $B=200 \mathrm{G}$.

known. The gradual distribution of switching current is either due to spin-polarized current or self-field of ferro- magnetic Co layer. The spins can penetrate through the mesa and scattering might decrease the degree of polarization. The first two branches cannot be seen in Fig. 2a, presumably first a few layers just underneath Au film are not cleaved properly or inhomogeneous intercalation of the crystal might cause such kind of effect. In a set of Josephson junctions as we have, the weaker layers within the stack switch into the quasiparticle state earlier than the others. This makes impossible to identify a particular quasiparticle branch in the $I-V$ characteristics corresponding to a Josephson junction within the mesa. As seen in Fig. 2b, the application of $200 \mathrm{G}$ external magnetic field increases the gradient in quasiparticle branches since the field increases the polarization of the current. However, the field does not affect branches observed at high bias, presumably, the injected spin-polarized quasiparticles through the multilayer begin to loose their spin-direction memory when they reach to some $\mathrm{CuO}_{2}$ planes far away from the ferromagnetic layer. Increasing magnetic field reduces the magnitude of switching current and hysteretic quasiparticle branches diminish above $700 \mathrm{G}$, only return branches persist.

The influence of spin-polarized current on IJJ tunneling characteristics indicates that critical current is smaller at the $\mathrm{CuO}_{2}$ sheets close to the ferromagnetic Co layer. Magnetic field dependence of hysteretic quasiparticle branches of the mesas with Co shows different behavior than the samples without ferromagnetic layer. The switching current for each branch in the mesa with ferromagnetic layer decreases with increasing magnetic field while it is constant for most of the scanned fields in mesa without ferromagnetic. Our study shows that the superconducting properties of $\mathrm{CuO}_{2}$ layers can be tuned with a ferromagnetic layer similar to inhomogeneous doping along the $c$-axis which can be used to generate $\mathrm{THz}$ radiation from Josephson vortices [4].

\section{Acknowledgement}

L.O. acknowledges support from the Turkish Academy of Sciences, in the framework of the Young Scientist Award Program (LO/TUBA-GEBIP/2002-1-17).

\section{References}

[1] D. Bang et al., Int. J. Mod. Phys. B 19 (2005) 1999.

[2] C. Kurter, L. Ozyuzer, J. Opt. Adv. Mater. 7 (2005) 415.

[3] H.S. Shin, H.J. Lee, Physica C 408-410 (2004) 623.

[4] S. Savelev et al., Phys. Rev. B 72 (2005) 144515. 